\title{
ARTICLE
}

Received 23 May 2014 | Accepted 13 Jun 2014 | Published 9 Jul $2014 \quad$ DOl: 10.1038/ncomms5409

\section{Merotelic attachments allow alignment and stabilization of chromatids in meiosis II oocytes}

\author{
Anna Kouznetsova ${ }^{1}$, Abrahan Hernández-Hernández ${ }^{1} \&$ Christer Höög ${ }^{1}$
}

The chromosome segregation process in human oocytes is highly error-prone, generating meiosis II (MII) oocytes with unbalanced chromatids that contribute to aneuploidy in offspring. This raises questions regarding the mechanism for transmission of chromatids and how chromatids evade the error correction mechanisms in Mll oocytes. Here, we analyse the behaviour of chromatids in mouse MII oocytes. We find that chromatids align at the spindle equator at the metaphase stage of MII and that their presence does not obstruct entry into the anaphase stage. The alignment process is mediated by merotelic (bi-directional) microtubule-kinetochore attachments, revealing a multi-domain organization of the kinetochore of mammalian meiotic chromosomes. Our results suggest that biorientation of chromatids stabilize microtubule attachments at the kinetochores in a tension-dependent manner. Our results also suggest that merotelic attachments contribute to chromosome mis-segregation in wild-type MII oocytes. Thus, merotely is an important promoter of aneuploidy in mammalian oocytes.

\footnotetext{
${ }^{1}$ Department of Cell and Molecular Biology, Karolinska Institutet, Berzelius väg 35, 17177 Stockholm, Sweden. Correspondence and requests for materials should be addressed to C.H. (email: Christer.Hoog@ki.se).
} 
A n estimated $20 \%$ of the oocytes in young women are aneuploid, and this percentage increases with age ${ }^{1,2}$. Aneuploidy is a leading genetic cause of miscarriage, congenital birth defects and mental retardation. Most meiotic errors in human oocytes arise due to premature separation of sister chromatids at the first meiotic division, resulting in the formation of meiosis II (MII) oocytes that carry unbalanced (single) chromatids ${ }^{3,4}$. To better understand the mechanism through which unbalanced chromatids contribute to aneuploidy, we have taken advantage of a mouse model $\left(S y c p 3^{-1-}\right)$ in which $30 \%$ of the oocytes at the MII stage carry one or a few chromatids ${ }^{5}$. The chromatids in Sycp $3^{-1-}$ MII oocytes are generated following premature separation of sister chromatids at the first meiotic division and they contribute to the formation of aneuploid embryos ${ }^{5-8}$.

Here we show that chromatids in mouse MII oocytes attain bidirectional microtubule-kinetochore interactions that align them at the spindle equator. This merotelic configuration generates tension that could stabilize microtubules bound to the kinetochore of chromatids, effectively eluding surveillance mechanisms monitoring tension and attachment, and contributing to aneuploidy in mammalian gametes.

\section{Results}

Chromatids align at the spindle equator in MII oocytes. We initially examined the spatial localization of chromatids in cytostatic factor (CSF)-induced metaphase II-arrested mouse oocytes ${ }^{9}$. Wild type and Sycp $3^{-1-}$ MII oocytes were stained with Hoechst 33342 nuclear dye and analysed using cellular imaging microscopy. We found that the chromosomal material in CSFinduced metaphase II-arrested Sycp $3^{-I-}$ MII oocytes aligned at the spindle equator (Fig. 1a), a surprising result considering that more than $30 \%$ of the Sycp $3^{-1-}$ MII oocytes carry chromatids ${ }^{5}$. Further analysis of fixed CSF-induced metaphase II-arrested Sycp3-1- MII oocytes stained with 4',6-diamidino-2phenylindole (DAPI) and anti-centromeric antiserum (ACA) ACA to respectively label chromatin and centromeres confirmed this result. We found that $90 \%$ of the chromatids in Sycp $3^{-1-}$ MII oocytes localized at the spindle equator (Fig. 1b,c), and that $70 \%$ of the misaligned chromatids localized close to the spindle equator (Fig. 1d). Our results therefore show that chromatids, similar to chromosomes, align at the spindle equator at the metaphase II stage of meiosis in Sycp $3^{-1}$ oocytes.

Chromatid alignment is supported by merotelic attachments. What promotes equatorial alignment of chromatids at the metaphase II stage in Sycp3 $3^{-1-}$ oocytes? To answer this question, we analysed the nature of the microtubule-kinetochore interactions for chromosomes and chromatids in CSF-induced metaphase II-arrested Sycp $3^{-1-}$ oocytes. We found that chromosomes at the spindle equator displayed an amphitelic configuration (Fig. 1e), a geometric orientation promoted by opposing end-on microtubule attachments to the sister kinetochores of chromosomes $^{10-12}$. Occasionally, one of the two sister kinetochores of chromosomes were bi-directionally attached to microtubules, suggesting also the presence of merotelic attachments (Supplementary Fig. 1). Importantly, chromatids localized at the spindle equator displayed bi-directional attachments, in this situation involving a single kinetochore only, generating a merotelic configuration (Fig. 1e). Analysis of chromatids localized at an intermediary position in between the pole and the equator of the spindle (Fig. 1e, Supplementary Fig. 2) revealed that these also were in contact with microtubules, either through bi-directional attachments or through lateral surface interactions. Thus, our results strongly suggest that lateral interactions and merotelic attachments contribute to congression and biorientation of chromatids at the metaphase stage of MII in oocytes.

Chromatid attachments contribute to spindle assembly checkpoint silencing. End-on attachment of microtubules to the kinetochores of chromosomes reduces Mad2 levels at kinetochores and contributes to silencing of the spindle assembly checkpoint (SAC) in mitotic and meiotic cells ${ }^{13-15}$. To find out whether the level of Mad2 was different at the kinetochores of chromosomes and chromatids, Sycp $3^{-1-}$ oocytes arrested at the metaphase II stage were labelled using an antibody against Mad2. We found the relative intensity of the Mad2 signal at kinetochores to be identical for chromosomes and chromatids (Fig. 2a,c). Importantly, treatment of metaphase II-arrested oocytes with nocodazole (a spindle poison that depolymerizes microtubular filaments and abolishes their attachments to the kinetochores) resulted in a strongly increased Mad2 signal at the kinetochores of both chromatids and chromosomes (Fig. 2a,c). We also measured the relative intensity of the Mad2 signal at the kinetochores of chromosomes and chromatids in Sycp3-1oocytes that were first released from CSF-induced metaphase II arrest by adding strontium. Again, we found that the Mad2 signal had the same intensity on the kinetochores of both chromatids and chromosomes, both in the presence and in the absence of nocodazole (Fig. 2b,d). These results suggest that merotelic endon attachments to the kinetochores of chromatids silence Mad2signalling and contribute to SAC inactivation at the metaphase II stage in Sycp3 $3^{-1-}$ oocytes.

Lagging chromosomes at anaphase in wild-type MII oocytes. To further investigate the process through which chromatids congress and segregate in MII oocytes, we used live-cell timelapse microscopy to track individual chromosomes and chromatids throughout cell division in MII oocytes. Wild type and Sycp $3^{-1-}$ mice were crossed with a mouse strain that carries an integrated $\mathrm{H} 2 \mathrm{~B}-\mathrm{mCh}$ Crry transgene, generating female mice that expresses a fluorescent histone fusion protein in oocytes. H2BmCherry-expressing wild type and Sycp3-1- oocytes were released from CSF-induced metaphase II arrest by strontium and then monitored by live-cell imaging through the second meiotic division. We found that most wild-type MII oocytes underwent an error-free cell division process (Fig. 3a,b; Supplementary Videos 1-4). Further analysis revealed that approximately $20 \%$ of the wild-type MII oocytes displayed transient chromosome misalignments, errors that were corrected before anaphase onset (Fig. 3a,b). Importantly, 9\% of the wild-type MII oocytes contained lagging chromosomes at anaphase onset, suggesting that merotelic microtubule-kinetochore attachments contribute significantly to chromosome mis-segregation events in wild-type MII oocytes.

Segregation errors in MII oocytes with chromatids. We next monitored H2B-mCherry-expressing Sycp $3^{-1-}$ oocytes that first were released from CSF-induced metaphase II arrest by strontium and then monitored by live-cell imaging through the second meiotic division. We found that $S y c p 3^{-1-}$ MII oocytes, relative to wild-type MII oocytes, displayed a slightly increased level of transient misalignments, as well as a twofold increased level of laggards at anaphase onset (Fig. 3a,b). To better understand the nature of the transient misalignments, these were further studied in more detail in individual oocytes. We found that transient misalignments at different time points during the second meiotic division were present in a larger fraction of $S y c p 3^{-1-}$ oocytes compared with wild-type oocytes (Fig. 4a). The increased 
a

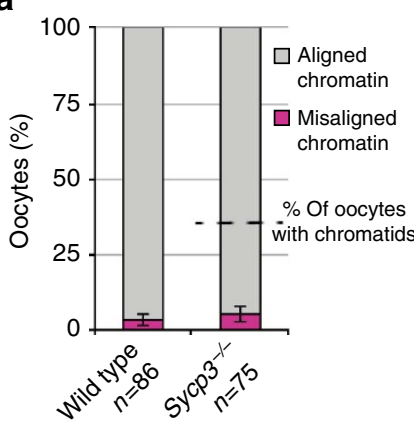

d
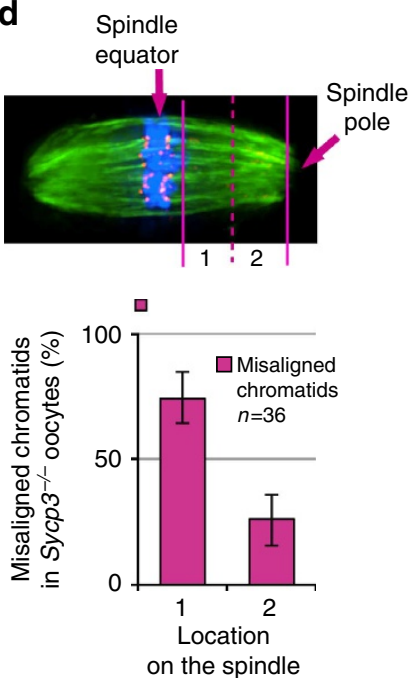

b
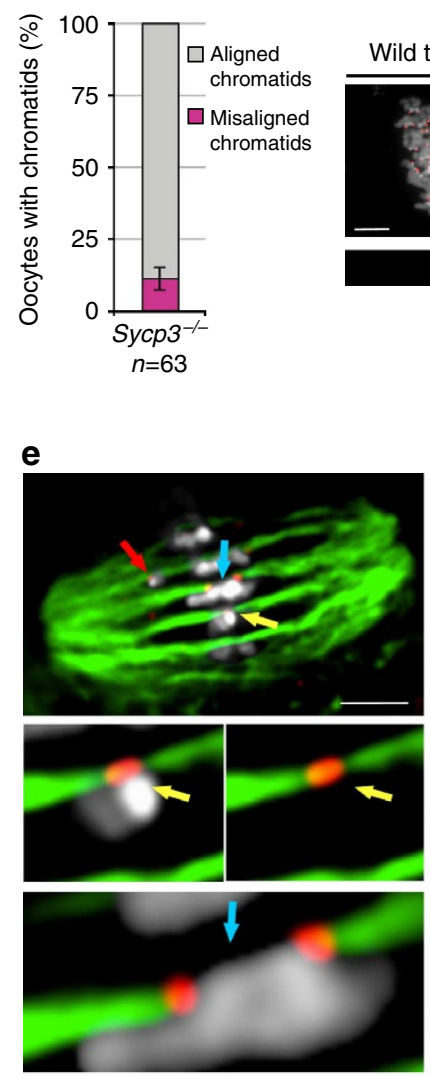

DAPI Tubulin

C
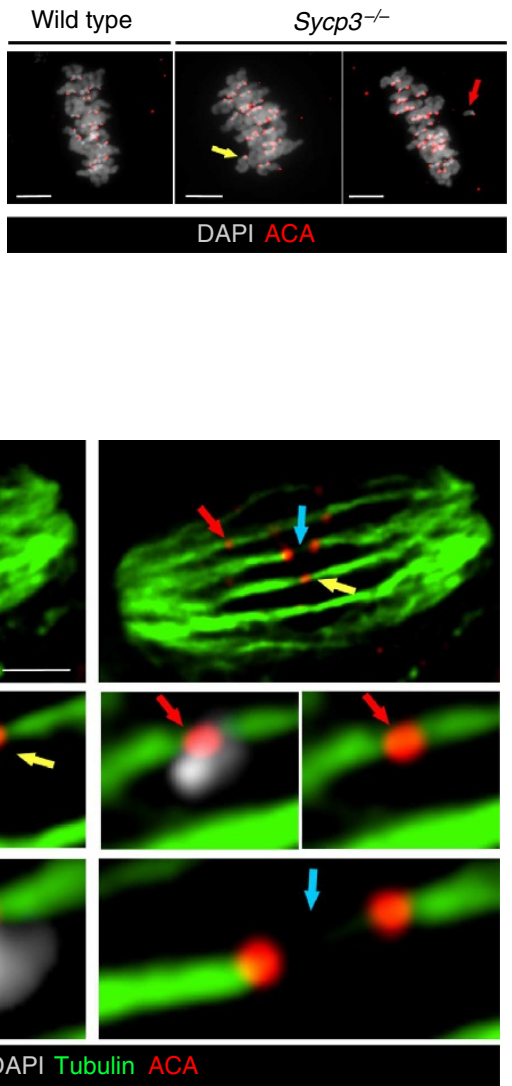

Figure 1 | Merotelic microtubule-kinetochore attachments promote equatorial alignment of chromatids. (a) Percentage of CSF-arrested meiosis II wild type and Sycp3 $3^{-/}$oocytes with chromatin (chromosomes or chromatids) aligned at the spindle equator or misaligned. Chromatin was labelled in vivo by Hoechst 33342 DNA staining. Over $30 \%$ of the Sycp3 $3^{-/-}$MII oocytes contained unbalanced chromatids (indicated by a dashed line ${ }^{5}$ ). Data collected from 11 independent experiments. (b) Chromatid localization in fixed MII CSF-arrested Sycp3-/- oocytes. Chromatids were scored as either aligned at the spindle equator or misaligned. Data from 16 independent experiments. (c) Localization of chromosomes and chromatids in fixed MII CSF-arrested wild type and Sycp3 ${ }^{-/-}$oocytes. Oocytes were stained with DAPI (DNA staining, grey) and ACA (centromere staining, red). Chromosomes in wild type and Sycp3 ${ }^{-/-}$oocytes localized to the spindle equator, whereas chromatids in Sycp3-/- oocytes were either aligned at the spindle equator (yellow arrow) or misaligned (red arrow). Images represent a single Z-plane, corresponding to the focal plane. (d) Localization of misaligned chromatids in fixed MII CSF-arrested Sycp3 ${ }^{-/}$oocytes. The distance between the outer edge of aligned chromatin at the spindle equator and the spindle pole was divided into two regions ( 1 and 2 ) and misaligned chromatids in Sycp3 ${ }^{-/-}$oocytes were then categorized into one of the regions. The results are shown as a percentage of all misaligned chromatids. Data from nine independent experiments. (e) Geometry for microtubule-kinetochore

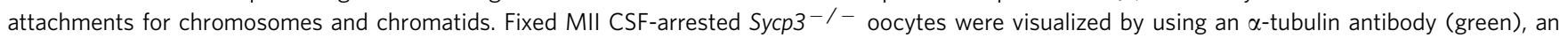
anti-centromeric ACA antibody (red) and DAPI to label chromatin (grey), following destabilization of dynamic non-kinetochore microtubules with calcium $^{26}$. Images shown represent a single Z-plane. Chromosomes (blue arrow) showed amphithelic attachments with microtubules that protruded into the sister centromeres from opposing sides. Aligned (yellow arrow) and misaligned (red arrow) chromatids displayed microtubules that connected to the single centromeres in a bi-directional (merotelic) manner, representing either end-on or lateral attachments. $n$ denotes the number of oocytes included in the experiments and vertical bars indicate s.d. (in $\mathbf{a}, \mathbf{b}$ and $\mathbf{d}$ ). Bars in $\mathbf{c}$ and $\mathbf{e}$ represent 10 and $5 \mu \mathrm{m}$, respectively.

presence of transient misalignments in Sycp3 $3^{-1-}$ MII oocytes was found to be owing to two cumulative factors: 1, that misalignments required more time for re-alignment at the equator (Fig. 4b), and 2, that misalignments occurred more frequently in a consecutive way in individual Sycp $3^{-1-}$ MII oocytes (Fig. 4c). At anaphase onset, $10 \%$ of $S y c p 3^{-/-}$MII oocytes retained misalignments - a segregation defect observed in approximately $2 \%$ of the wild-type MII oocytes (Fig. 3b). The increased presence of laggards and misalignments identified in Sycp3-I- MII oocytes (relative to wild-type MII oocytes) most likely relates to the presence of chromatids in mutant oocytes.

Chromatids do not delay entry into anaphase II. We then asked if the segregation defects in Sycp $3^{-1-}$ MII oocytes obstructed the ability of these cells to complete the second meiotic division. We found, however, that $S y c p 3^{-1-}$ MII oocytes entered into the anaphase stage with a similar temporal profile and as efficiently as wild-type oocytes (Fig. 3c,d). This result cannot be explained by the presence of an impaired SAC in Sycp3 $3^{-1-}$ MII oocytes, as nocodazole inhibited anaphase onset with similar efficiency in wild type and mutant oocytes (Fig. 3d). We conclude that neither the presence of single chromatids nor an increased level of segregation errors affected the ability of $S y c p 3^{-I-}$ MII oocytes to complete the second meiotic division.

\section{Discussion}

We show here that a large majority of the chromatids in Sycp $3^{-1-}$ MII oocytes align at the spindle equator at the 
a
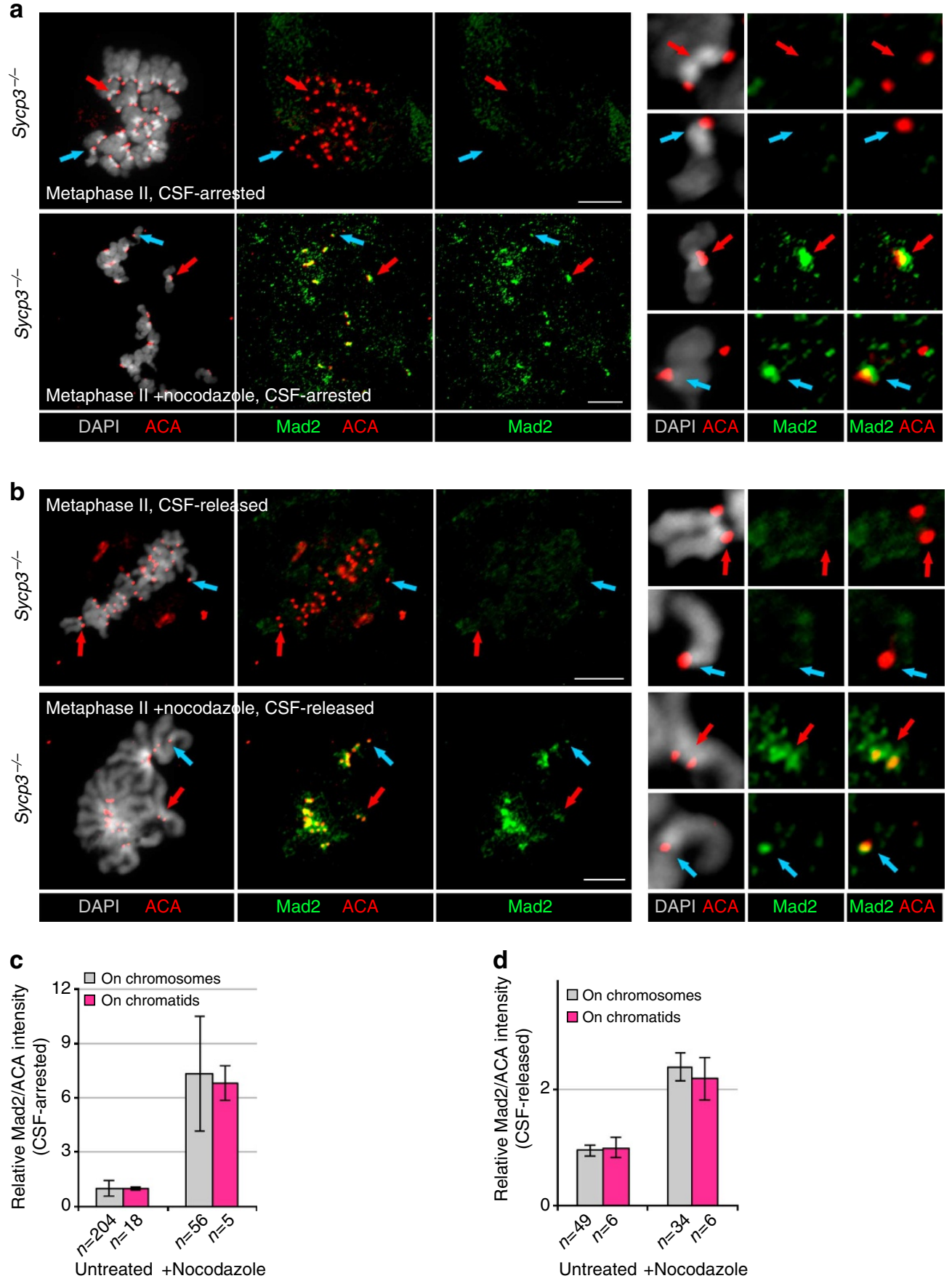

Figure 2 | Mad2 levels at the kinetochores of chromatids and chromosomes are identical. (a,b) Mad2 localization at kinetochores of chromosomes and chromatids was analysed in fixed Sycp3 $3^{-/}$CSF-arrested MII oocytes (a) or in Sycp3 $3^{-/}$metaphase II oocytes released from CSF arrest (90 min after addition of strontium) (b). Staining was performed in the presence or absence of nocodazole. Mad2 was labelled using an antibody (green), centromeres labelled by an ACA antibody (red) and chromatin stained by DAPI (grey). Individual representative chromosomes and chromatids (indicated by red and blue arrows, respectively) were separately magnified to visualize the Mad2 (green) and ACA (red) signals. Scale bars, $10 \mu \mathrm{m}$. (c,d) Relative Mad2 levels at kinetochores of chromosomes and chromatids were analysed in fixed Sycp $3^{-/}$- CSF-arrested MII oocytes (c) or in Sycp ${ }^{-/}$- MII oocytes released from CSF arrest (90 min after addition of strontium) (d). Mad2 levels were calculated from the relative intensity of the Mad2/ACA antibody signals at kinetochores of chromosomes (grey) and chromatids (red). Staining was performed in the presence or absence of nocodazole. The relative intensity values were normalized for the chromosomes to a value of $1 . n$ denotes the number of analysed chromosomes or chromatids, data collected from three independent experiments. Error bars indicate s.d.

metaphase stage, displaying merotelic microtubule-kinetochore attachments. This result is in agreement with previous studies of the behaviour of chromatids in mammalian somatic cells and in plant meiotic cells ${ }^{16-19}$. These studies showed that mammalian and plant kinetochores are organized into multiple domains, promoting the formation of bi-directional microtubule attachments at the kinetochores of chromatids ${ }^{17,18}$. An important question arising from these studies is why merotelic, and not monotelic, attachments are established at the kinetochores of chromatids? In both geometric orientations, the attachments to 


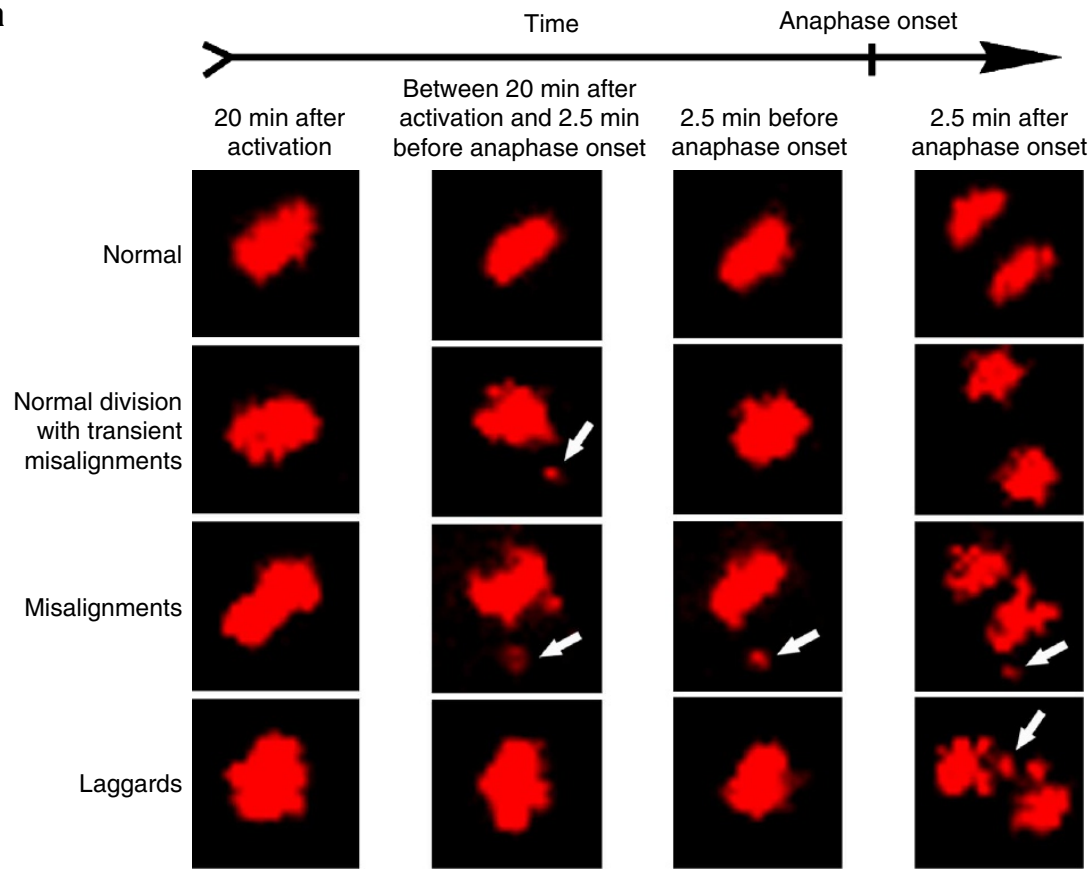

b

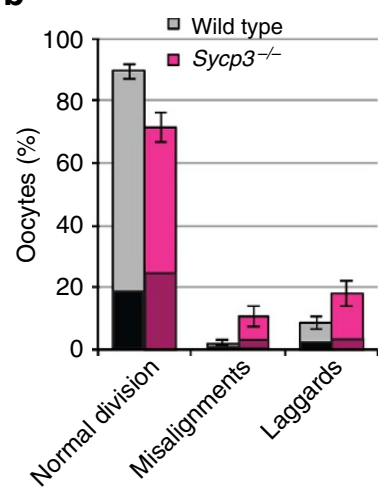

C

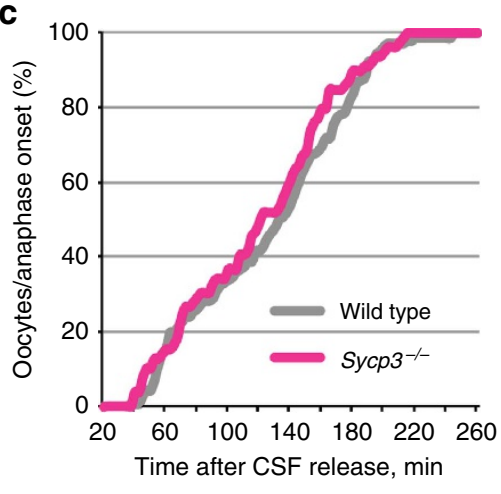

d

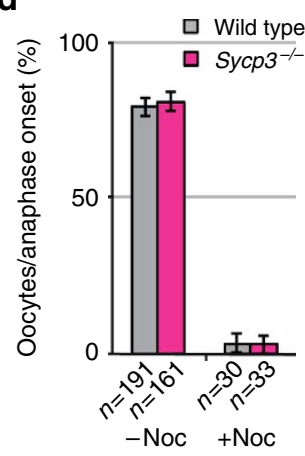

Figure 3 | Sycp3 $3^{-1-}$ oocytes with chromatids display an increased level of segregation defects, but their entry into anaphase is not obstructed. (a) Chromosome and chromatid segregation patterns in mouse MII CSF-released oocytes were identified by time-lapse imaging every 2.5 min. The columns from left to right show four different time points following CSF release. In a normal MII division (upper row), the chromosomes localized as a single chromatin structure at the metaphase plate and segregated into two separate chromatin structures. We observed in both wild type and Sycp $3^{-/-}$ metaphase II oocytes three types of deviating patterns, including transient misalignments (second row), misalignments that persisted at anaphase onset (third row) and chromatin that remained at the spindle midzone at anaphase onset (laggards, bottom row). Arrows indicate chromatin fragments, corresponding to chromosomes or chromatids. (b) Quantification of chromosome and chromatid segregation patterns in mouse oocytes. Time-lapse imaging was performed for 150 wild type (grey) and 84 Sycp $3^{-/-}$(red) MII oocytes following release from CSF arrest and the segregation pattern for individual cells categorized into one of three categories. Transient misalignments observed in cells categorized into one of the three categories are shown in black (for wild-type oocytes) or in dark red (for Sycp3 $3^{-/-}$oocytes). Data from seven independent experiments. Error bars indicate s.d. (c) Cumulative curves for the time of anaphase onset in wild type (grey line, $n=150$ ) and Sycp3 ${ }^{-/-}$(red line, $n=84$ ) MII oocytes, released from CSF arrest. The vertical axis shows the cumulative percentage of all oocytes that entered anaphase, while the horizontal axis indicates the time after CSF release, in minutes. Data from seven independent experiments. (d) Efficiency in entering anaphase. Wild type and Sycp3 ${ }^{-/-}$MII oocytes were released from CSF arrest in the presence or absence of nocodazole and monitored by live-cell imaging. The percentage of wild type (grey bars) and Sycp $3^{-/-}$(red bars) MII oocytes that entered anaphase was identical. Nocodazole blocked anaphase onset for both wild type (grey bars) and Sycp $3^{-/-}$(red bars) MII oocytes. $n$ denotes the number of oocytes included in the experiments. Error bars indicate s.d. Data from three independent experiments.

the kinetochores should contribute to the silencing of the $\mathrm{SAC}^{19,20}$. We find, however, very rarely chromatids to be localized to the spindle poles in Sycp $3^{-/}$MII oocytes, which would be the predicted localization of chromatids resulting from a monopolar attachment. Syntelic attachments (analogous to the monopolar attachments for chromatids) are effectively replaced by amphitelic attachments (analogous to the merotelic attachments for chromatids) in somatic cells during mitosis ${ }^{19,20}$ This is likely a consequence of the fact that amphitelic, but not syntelic attachments, generates intra- and inter-kinetochore tension, tension that increases the number of microtubules bound to kinetochores and stabilize their interactions ${ }^{21-23}$. We propose that the merotelic attachments observed at chromatids in Sycp3 $3^{-/-}$MII oocytes fulfil a role similar to that of amphitelic 
a

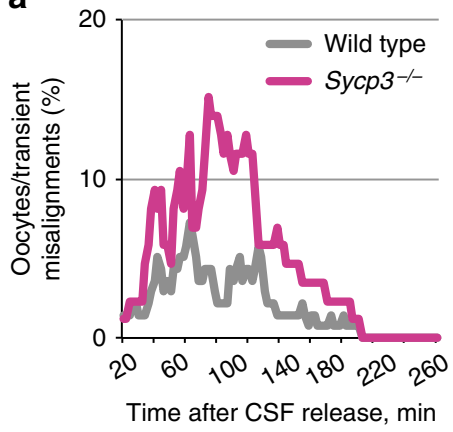

b

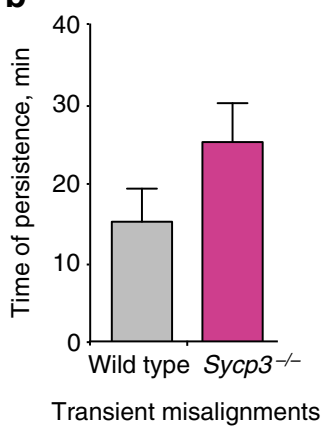

C

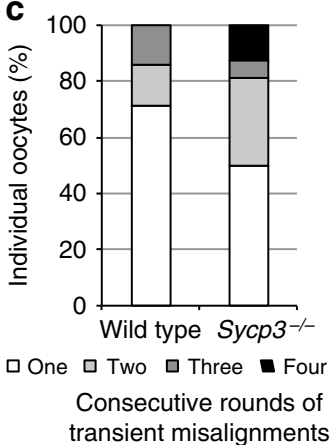

Figure 4 | Transient misalignments take place at an increased rate and remain uncorrected for longer period of time in Sycp3 ${ }^{-/-}$CSF-released MII oocytes. (a) Transient misalignments are frequently observed in Sycp3-/- MIl oocytes. Occurrence of transient misalignments in wild type (grey line) and Sycp3 $3^{-/-}$(red line) MII oocytes following the release from CSF arrest, shown as percentage of all oocytes that initiated anaphase. The vertical axis shows the percentage of oocytes that displayed transient misalignments at each time point, while the horizontal axis indicates the time after release from CSF arrest, in minutes. (b) Transient misalignments persist longer in Sycp3 ${ }^{-/-}$oocytes. Persistence of transient misalignments was measured in wild type and Sycp3 $3^{-/-}$MII oocytes following the release from CSF arrest $(P=0.03$, Mann-Whitney test). The average time of persistence for transient misalignments was $15 \mathrm{~min}$ in wild-type oocytes (grey column, $n=31$ ), versus 25 min for Sycp3 ${ }^{-/-}$oocytes (red column, $n=23$ ). Vertical axis shows time in minutes, error bars indicate s.e.m. (c) Transient misalignments appear in a consecutive manner in individual Sycp3-/- oocytes. The number of sequential transient misalignments was determined in wild type $(n=28)$ and Sycp $3^{-/-}(n=17)$ MII oocytes following the release from CSF arrest. The grey scale within the columns indicates the number of consecutive rounds that transient misalignments appeared in individual oocytes. Data from seven independent experiments.

attachments for chromosomes, generating tension across the single kinetochore of chromatids and contributing to microtubule recruitment and stabilization. Formation of merotelic attachments at chromatids could be of particular importance in acentriolar meiotic cells, where tension at kinetochores cannot be established through polar ejection forces ${ }^{24-26}$. The biorientation process of chromatids in Sycp3 $3^{-/-}$MII oocytes is likely to be promoted by lateral gliding of chromatids towards the spindle equator, as shown for somatic cells ${ }^{24-26}$. The fact that we see very few chromatids close to the poles in $S y c p 3^{-/-}$MII oocytes strongly suggests that tension, resulting from merotelic attachments, effectively contributes to alignment and stabilization of chromatids at the spindle equator. Tension established though bi-directional attachment of chromatids should help to maintain SAC inactivation and to elude surveillance mechanism monitoring attachment and tension, promoting chromatid transmission and formation of aneuploid gametes. The CSF arrest that precedes activation and anaphase entry in MII oocytes could provide time for establishing merotelic attachments for chromatids, as SAC is not essential for maintaining CSF arrest in mouse oocytes ${ }^{9}$. Importantly, we have found that also wild-type MII oocytes frequently display merotelic attachments at the metaphase stage, as well as lagging chromosomes at anaphase onset. This strongly suggests that merotelic attachments contribute significantly to chromosome mis-segregation in wild-type female germ cells, similar to what previously been documented for somatic cells during mitosis ${ }^{10,11,27}$. In conclusion, our results show that merotely contributes to aneuploidy in mammalian oocytes.

\section{Methods}

Animal studies. The animal experiments were approved by the Stockholm-North Animal Ethical Committee. Oocytes were taken from 3-4-week or 11-13-week old female mice carrying a null mutation for the $S y c p 3$ gene and their wild-type siblings, produced on the mixed C57BL/6NCrl $\times 129 /$ OlaIHsd background 6 . Ovaries from wild type and Sycp3 $3^{-/}$mice were dissected and the oocytes at the germinal vesicle stage were released with the $26 \mathrm{G}$ needle into M2 medium (Sigma). Oocytes that underwent germinal vesicle breakdown within $2 \mathrm{~h}$ were further matured for $13-14 \mathrm{~h}$ at $37^{\circ} \mathrm{C}$. Oocytes that extruded the polar body and arrested in a CSFdependent manner at the metaphase II stage were released from cumulus cells by pipetting and used for further studies. Oocytes were activated through incubation in $\mathrm{Ca}^{2+}$-free potassium simplex optimization medium (KSOM) media supplemented with $10 \mathrm{mM} \mathrm{SrCl}_{2}$. For visualization of chromatin alignment, live oocytes were stained with $1 \mu \mathrm{g} \mathrm{ml}^{-1}$ Hoechst 33342 (Invitrogen) and images captured at $\times 10$ magnification on a Leica DMRXA microscope.

Immunofluorescence microscopy. Cumulus-free CSF-arrested were fixed immediately following their collection, and activated oocytes were fixed $90 \mathrm{~min}$ after their release into strontium-containing activation medium. In brief, oocytes were incubated $3 \mathrm{~min}$ in Tyrode's solution (Sigma) to remove zone pellucida and dropped on a slide dipped in $1 \%$ paraformaldehyde $/ 0.15 \%$ Tritoin X-100. The slides were air-dried for $2 \mathrm{~h}$, rehydrated in phosphate-buffered saline (PBS) $/ 0.01 \%$ Triton X-100 and then blocked for $1 \mathrm{~h}$ in $3 \%$ bovine serum albumin in PBS $/ 0.01 \%$ Triton X-100. In total, $400 \mathrm{nM}$ nocodazole (Sigma) was added to the M2 media or to the $\mathrm{Ca}^{2+}$-free $\mathrm{KSOM}$ medium with $\mathrm{SrCl}_{2}$ for $60-100 \mathrm{~min}$. Visualization of microtubule-kinetochore geometry was done in intact CSF-arrested oocytes. Microtubules were destabilized in $\mathrm{Ca}^{2+}$ buffer $(100 \mathrm{mM}$ PIPES pH 7.0, $1 \mathrm{mM}$ $\mathrm{MgCl}_{2}, 0.1 \mathrm{mM} \mathrm{CaCl}_{2}, 0.1 \%$ Triton X-100) for $90 \mathrm{~s}$ and fixed with $1 \%$ formaldehyde in the $\mathrm{Ca}^{2+}$ buffer for $30 \mathrm{~min}$ at room temperature. After the fixation, the oocytes were washed with PBS $/ 0.1 \%$ Triton X-100 four times, extracted overnight at $4{ }^{\circ} \mathrm{C}$, and then blocked with 3\% bovine serum albumin in PBS/0.01\% Triton X-100 for $1 \mathrm{~h}$ at room temperature. The following antibodies and dilutions were used for immunolocalization studies: human ACA antiserum 1:200 (Antibodies, Cat\# 15-234-0001), rabbit anti-MAD2 1:50 (Covance, Cat \#PRB-452C) and mouse anti- $\alpha$-tubulin 1:200 (Sigma, Cat\# T-9026). Secondary antibodies used were swineanti-rabbit FITC (DAKO) 1:400, donkey anti-mouse Alexa488 (Invitrogen) 1:1,000, goat-anti-human Cy5 (Amersham) 1:3,000 and goat-anti-human Cy3 (Amersham) 1:1,000. Spread oocytes after staining were mounted in Prolong Gold mounting medium containing DAPI (Molecular Probes) and a single image representing the focal plane was captured using Leica DMRXA microscope at $\times 1,000$ magnification. The entire spindle in fixed intact oocytes was imaged at $\times 1,000$ magnification every $0.2 \mu \mathrm{m}$ using a DeltaVision microscope (Applied Precision). Images were processed using Openlab 3.1.4 software (Improvision), Volocity 6.0 software (Perkin Elmers), SoftWoRx 3.3.1 software (DeltaVision) and Adobe Photoshop 8.0. For quantification of signals on the kinetochores, the centromeric regions as defined by ACA signal were outlined and the integrated density of Mad2 and ACA signals on single chromatids and chromosomes were calculated using ImageJ software. The average ratio Mad2/ACA at chromosomes without nocodazole were normalized to 1 and thereafter compared with the values for the chromatids. To minimize the variability in quantification of following nocodazole treatment, oocytes incubated with and without nocodazole were fixed on the same slide.

Time-lapse imaging. A reporter gene, coding for histone $\mathrm{H} 2 \mathrm{~B}$ fused to a mCherry fluorescent molecule, was introduced into the experimental mouse strain by backcrossing with reporter mice carrying a H2B-mCherry fusion gene ${ }^{28}$. Timelapse imaging of oocytes undergoing second meiotic division was performed in 
$\mathrm{Ca}^{2+}$-free $\mathrm{KSOM}$ medium supplemented with $10 \mathrm{mM}$ of $\mathrm{SrCl}_{2}$ using a Leica DMI6000 microscope at $37^{\circ} \mathrm{C}, 5 \% \mathrm{CO}_{2}$. Red fluorescence and differential contrast (DIC) images were captured every $2.5 \mathrm{~min}$ at $\times 20$ magnification for $4 \mathrm{~h}$, for a total of 20-25 $1.5-\mu \mathrm{m} \mathrm{Z}$-sections. For the nocodazole assay, oocytes were imaged in the

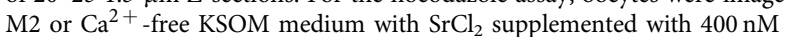
nocodazole (Sigma) for $4 \mathrm{~h}$. Oocytes that degraded during that period were excluded from further analysis. The first time frame when chromatin showed a bidirectional poleward movement was scored as anaphase onset. Misalignments in oocytes were scored when smaller chromatin bodies became visibly separated from the central chromatin mass. Experiments involving Sycp $3^{-/-}$oocytes were always performed in parallel with wild-type oocytes.

Statistical analysis. Statistical analysis was performed using Prism 6 software.

\section{References}

1. Handel, M. A. \& Schimenti, J. C. Genetics of mammalian meiosis: regulation, dynamics and impact on fertility. Nat. Rev. Genet. 11, 124-136 (2010).

2. Nagaoka, S. I., Hassold, T. J. \& Hunt, P. A. Human aneuploidy: mechanisms and new insights into an age-old problem. Nat. Rev. Genet. 13, 493-504 (2012).

3. Angell, R. First-meiotic-division nondisjunction in human oocytes. Am. J. Hum. Genet. 61, 23-32 (1997).

4. Handyside, A. H. Molecular origin of female meiotic aneuploidies. Biochim. Biophys. Acta 1822, 1913-1920 (2012)

5. Kouznetsova, A., Lister, L., Nordenskjold, M., Herbert, M. \& Hoog, C. Bi-orientation of achiasmatic chromosomes in meiosis I oocytes contributes to aneuploidy in mice. Nat. Genet. 39, 966-968 (2007).

6. Yuan, L. et al. Female germ cell aneuploidy and embryo death in mice lacking the meiosis-specific protein SCP3. Science 296, 1115-1118 (2002)

7. Lightfoot, D. A., Kouznetsova, A., Mahdy, E., Wilbertz, J. \& Hoog, C. The fate of mosaic aneuploid embryos during mouse development. Dev. Biol. 289, 384-394 (2006).

8. Wang, H. \& Hoog, C. Structural damage to meiotic chromosomes impairs DNA recombination and checkpoint control in mammalian oocytes. J. Cell Biol. 173, 485-495 (2006).

9. Tsurumi, C., Hoffmann, S., Geley, S., Graeser, R. \& Polanski, Z. The spindle assembly checkpoint is not essential for CSF arrest of mouse oocytes. J. Cell Biol. 167, 1037-1050 (2004).

10. Walczak, C. E., Cai, S. \& Khodjakov, A. Mechanisms of chromosome behaviour during mitosis. Nat. Rev. Mol. Cell Biol. 11, 91-102 (2010).

11. Gregan, J., Polakova, S., Zhang, L., Tolic-Norrelykke, I. M. \& Cimini, D. Merotelic kinetochore attachment: causes and effects. Trends Cell Biol. 21, 374-381 (2011).

12. Watanabe, Y. Geometry and force behind kinetochore orientation: lessons from meiosis. Nat. Rev. Mol. Cell Biol. 13, 370-382 (2012).

13. Kallio, M., Eriksson, J. E. \& Gorbsky, G. J. Differences in spindle association of the mitotic checkpoint protein Mad2 in mammalian spermatogenesis and oogenesis. Dev. Biol. 225, 112-123 (2000).

14. Homer, H. A. et al. Mad2 prevents aneuploidy and premature proteolysis of cyclin B and securin during meiosis I in mouse oocytes. Genes Dev. 19, 202-207 (2005).

15. Foley, E. A. \& Kapoor, T. M. Microtubule attachment and spindle assembly checkpoint signalling at the kinetochore. Nat. Rev. Mol. Cell Biol. 14, 25-37 (2013).

16. Rieder, C. L., Davison, E. A., Jensen, L. C., Cassimeris, L. \& Salmon, E. D. Oscillatory movements of monooriented chromosomes and their position relative to the spindle pole result from the ejection properties of the aster and half-spindle. J. Cell Biol. 103, 581-591 (1986).
17. Khodjakov, A., Cole, R. W., McEwen, B. F., Buttle, K. F. \& Rieder, C. L. Chromosome fragments possessing only one kinetochore can congress to the spindle equator. J. Cell Biol. 136, 229-240 (1997).

18. Yu, H. G. \& Dawe, R. K. Functional redundancy in the maize meiotic kinetochore. J. Cell Biol. 151, 131-142 (2000).

19. O'Connell, C. B. et al. The spindle assembly checkpoint is satisfied in the absence of interkinetochore tension during mitosis with unreplicated genomes. J. Cell Biol. 183, 29-36 (2008).

20. Rieder, C. L., Cole, R. W., Khodjakov, A. \& Sluder, G. The checkpoint delaying anaphase in response to chromosome monoorientation is mediated by an inhibitory signal produced by unattached kinetochores. J. Cell Biol. 130, 941-948 (1995).

21. Akiyoshi, B. et al. Tension directly stabilizes reconstituted kinetochoremicrotubule attachments. Nature 468, 576-579 (2010).

22. Khodjakov, A. \& Pines, J. Centromere tension: a divisive issue. Nat. Cell Biol. 12, 919-923 (2010)

23. Maresca, T. J. \& Salmon, E. D. Welcome to a new kind of tension: translating kinetochore mechanics into a wait-anaphase signal. J. Cell Sci. 123, 825-835 (2010).

24. Maro, B., Howlett, S. K. \& Webb, M. Non-spindle microtubule organizing centers in metaphase II-arrested mouse oocytes. J. Cell Biol. 101, 1665-1672 (1985).

25. Nicklas, R. B., Waters, J. C., Salmon, E. D. \& Ward, S. C. Checkpoint signals in grasshopper meiosis are sensitive to microtubule attachment, but tension is still essential. J. Cell Sci. 114, 4173-4183 (2001).

26. Kitajima, T. S., Ohsugi, M. \& Ellenberg, J. Complete kinetochore tracking reveals error-prone homologous chromosome biorientation in mammalian oocytes. Cell 146, 568-581 (2011).

27. Cimini, D. et al. Merotelic kinetochore orientation is a major mechanism of aneuploidy in mitotic mammalian tissue cells. J. Cell Biol. 153, 517-527 (2001)

28. Abe, T. et al. Establishment of conditional reporter mouse lines at ROSA26 locus for live cell imaging. Genesis 49, 579-590 (2011).

\section{Acknowledgements}

This work was supported by grants from the Swedish Cancer Society, the Swedish Research Council, Torsten and Ragnar Söderbergs Stiftelser and Karolinska Institutet. We thank Sonata Valentiniene for technical support.

\section{Author contributions}

A.K. performed most of the experiments. A.H.-H. contributed to the analysis of microtubule-kinetochore interactions. A.K. and C.H. initiated and finalized the study. A.K. and C.H. wrote the paper. All authors analysed data, discussed the results and commented on the paper.

\section{Additional information}

Supplementary Information accompanies this paper at http://www.nature.com/ naturecommunications

Competing financial interests: The authors declare no competing financial interests.

Reprints and permission information is available online at http://npg.nature.com/ reprintsandpermissions/

How to cite this article: Kouznetsova, A. et al. Merotelic attachments allow alignment and stabilization of chromatids in meiosis II oocytes. Nat. Commun. 5:4409 doi: 10.1038/ncomms5409 (2014). 